\title{
ORIGIN OF PURE SPIN SUPERRADIANCE
}

\author{
V.I.Yukalov \\ Department of Mathematics and Statistics \\ Queen's University, Kingston, Ontario K'7L 3N6, Canada \\ and \\ Bogolubov Laboratory of Theoretical Physics \\ Joint Institute for Nuclear Research, Dubna 141980, Russia
}

70.20.+q; 76.60.-k; 76.60.Es

Typeset using REVTEX 


\begin{abstract}
The question addressed in this paper is: What originates pure spin superradiance in a polarized spin system placed inside a resonator? The term "pure" means that no initial coherence is imposed on spins, and its appearance manifests a purely self-organized collective effect. The consideration is based on a microscopic model with dipole spin interactions. An accurate solution of evolution equations is given. The results show that the resonator Nyquist noise does not play, contrary to the common belief, any role in starting spin superradiance, but the emergence of the latter is initiated by local spin fluctuations. The decisive role of nonsecular dipole interactions is stressed.
\end{abstract}

A polarized spin system prepared in a nonequilibrium state returns to equilibrium through the spin-spin and spin-lattice relaxation mechanisms. The spin relaxation can be drastically accelerated if the nonequilibrium magnetic system is placed inside a coil of a resonance electric circuit with the natural frequency tuned to the precession frequency of spin magnetic moments [1]. The strong shortening of the relaxation time is caused by the coherence between individual rotating spins, which develops as a result of the interaction between the rotating magnetization and the resonator feedback field. This coherent phenomenon is analogous to the Dicke superradiance [2] occurring in atomic and molecular systems, and so Bloembergen and Pound [1] also called this fast collective damping in spin systems the radiation damping. Friedberg and Hartmann [3] noted that, in fact, the whole process in spin systems involves no radiation at all, but merely nonradiative transfer of energy from the sample to the coil and back, the energy being dissipated in the circuit ohmically. And what one measures in experiments with spin systems is not the intensity of radiation, but the power of electric current. Nevertheless, the term superradiance has been accepted for the transient coherent phenomenon in spin systems, when it develops as a self-organized process, similarly to the Dicke superradiance. The similarity between the 
latter and the spin superradiance is not the sole excuse for the accepted term. Another reason is to distinguish the spin superradiance from nuclear induction, free or collective, that is also a coherent phenomenon although not self-organized, but forced by thrusting onto a sample an initial coherence. One more justification for using the term spin superradiance is that this effect is always accompanied by coherent magneto-dipole radiation, though the corresponding radiation intensity is very weak as compared to the easily measured current power [4].

The term pure spin superradiance is used in order to stress that this is a purely selforganized process, when coherence develops from an absolutely incoherent state. This is to be distinguished from triggered spin superradiance during which collective effects also play an important role, but the process starts from a coherent initial state, so that the imposed initial coherence triggers the development of a correlated state, in the same way as triggered optical superradiance [5] happens in atomic and molecular systems. When spin superradiance is caused by nuclear spins, it can be called the nuclear spin superradiance. A system of ion spins in a resonator cavity can, in principle, be also a source of spin superradiance.

The nuclear spin superradiance has been recently observed in a series of experiments [611] with different substances: from $\mathrm{Al}$ nuclear spins in ruby $\left(\mathrm{Al}_{2} \mathrm{O}_{3}\right)$ and from proton spins in propanediol $\left(\mathrm{C}_{3} \mathrm{H}_{8} \mathrm{O}_{2}\right)$, butanol $\left(\mathrm{C}_{4} \mathrm{H}_{9} \mathrm{OH}\right)$, and ammonia $\left(\mathrm{NH}_{3}\right)$. The interpretation of the pure spin superradiance in these experiments was based on the following picture. A system of polarized spins is placed in a constant magnetic field directed opposite to the sample polarization. This means that the spin system is prepared in an inverted state. The sample is put inside the coil of a passive electric circuit whose natural frequency is tuned to the Zeeman frequency of spins. Fluctuating magnetic field formed by the thermal Nyquist noise of the resonance circuit starts moving spins from their position of unstable equilibrium. The motion of spins induces electric current in the circuit, which creates a stronger magnetic field acting back on spins. Under the action of the feedback field, spins move faster increasing even more the resonator feedback field, and so on. This avalanche-type process results in a fast spin relaxation. Such is the commonly accepted picture of pure spin superradiance. 
However in this, generally correct, picture there is one suspicious point, namely, that the beginning of the process is originated by the thermal Nyquist noise of resonator. Really, if one attentively reads the classical paper by Bloembergen and Pound [1], then one finds there the estimate for the thermal damping, due to the thermal noise in resonator, showing that this damping is so negligibly small for macroscopic systems that it can never produce the initial thermal relaxation.

Thus we confront the alternative: either the common belief that this is the resonator thermal noise which initiates the pure spin superradiance is a delusion or Bloembergen and Pound are wrong. To resolve this paradox and to answer the question "what actually is the origin of pure spin superradiance" is the aim of the present paper.

The solution of the formulated problem meets the following difficulty. As follows from the analysis of Bloembergen and Pound [1], the homogeneous approach provided by the Bloch equation is not sufficient for correctly describing the process, but inhomogeneous local fields, that produce a microscopic relaxation mechanism, are essential. The phenomenological Bloch equation, even being solved in a reasonably accurate approximation [12], can describe only the triggered spin superradiance, when an initial coherence is imposed by assuming nonzero initial conditions for transverse magnetization. To take into account inhomogeneous local fields providing a microscopic relaxation mechanism means the necessity of dealing with a microscopic model. Writing the equations of motion for spin components we get a $3 N$-dimensional system of nonlinear differential equations for $N$ spins, plus the Kirchhoff equation for an electric circuit. If one invokes any approach, to solve this system of equations, based on the uniform mean-field approximation, then one immediately returns to a homogeneous picture equivalent to the Bloch equation, thus loosing an information on local fields. When the number of spins, $N$, is not too large, say $N \sim 10-10^{3}$, then it is possible to resort to numerical calculations. Such a computer simulation has been done [4] (for mathematical details see [13]) and has been shown that, really, the pure spin superradiance can exist without the resonator Nyquist noise. However, such computer simulations have the following deficiencies: (i) they are time consuming; (ii) they are able to give only 
a qualitative description, since the number of spins involved is incomparably smaller than what one has in real samples with $N \sim 10^{23}$; (iii) they do not give analytical formulas that would be convenient to study with respect to the variation of all, sometimes numerous, parameters characterizing the system. Therefore, computer simulations can give a feel of what is happening, but cannot provide decisive answers. Below an analytical solution of microscopic equations is presented.

A system of nuclear spins is described [14] by the Hamiltonian

$$
\hat{H}=\frac{1}{2} \sum_{i \neq j}^{N} H_{i j}-\mu \sum_{i=1}^{N} \vec{B} \vec{S}_{i}
$$

with dipole spin interactions

$$
H_{i j}=\frac{\mu^{2}}{r_{i j}}\left[\vec{S}_{i} \vec{S}_{j}-3\left(\vec{S}_{i} \vec{n}_{i j}\right)\left(\vec{S}_{j} \vec{n}_{i j}\right)\right],
$$

where $\mu$ is a nuclear magneton and

$$
\vec{n}_{i j} \equiv \frac{\vec{r}_{i j}}{r_{i j}}, \quad \vec{r}_{i j} \equiv \vec{r}_{i}-\vec{r}_{j}, \quad r_{i j} \equiv\left|\vec{r}_{i j}\right| .
$$

The total magnetic field

$$
\vec{B}=H_{0} \vec{e}_{z}+H \vec{e}_{x}
$$

consists of a constant external field $H_{0}$ and an alternating field $H$ of a resonator coil. The latter has $n$ turns of a cross-section area $A_{c}$ over a length $l$. The resonance electric circuit includes a resistance $R$, inductance $L$, and capacity $C$. The alternating resonator field

$$
H=\frac{4 \pi n}{c l} j
$$

is formed by an electric current satisfying the Kirchhoff equation

$$
L \frac{d j}{d t}+R j+\frac{1}{C} \int_{0}^{t} j(\tau) d \tau=-\frac{d \Phi}{d t}+E_{f}
$$

in which $E_{f}$ is an electromotive force and

$$
\Phi=\frac{4 \pi}{c} n A_{c} \eta \rho \frac{\mu}{N} \sum_{i=1}^{N}\left\langle S_{i}^{x}\right\rangle
$$


is a magnetic flux through the coil; $\eta \equiv V / V_{c}$ being a filling factor; $V_{c} \equiv l A_{c}$, the coil volume; $\rho \equiv N / V$, density of spins.

Define the resonator natural frequency $\omega \equiv 1 / \sqrt{L C}$, ringing width $\gamma_{3} \equiv R / 2 L$, and dimensionless fields

$$
h \equiv \frac{\mu H}{\hbar \gamma_{3}}, \quad f \equiv \frac{c \mu E_{f}}{n A_{c} \hbar \gamma_{3}^{2}} .
$$

Introduce the parameter

$$
\alpha_{0} \equiv \pi \eta \frac{\rho \mu^{2}}{\hbar \gamma_{3}}
$$

characterizing the strength of coupling between the spin system and the resonator. Let us also use the notation

$$
u \equiv \frac{1}{N} \sum_{i=1}^{N}\left\langle S_{i}^{-}\right\rangle, \quad s \equiv \frac{1}{N} \sum_{i=1}^{N}\left\langle S_{i}^{z}\right\rangle
$$

for the mean spin components, where $\langle\ldots\rangle$ implies statistical averaging. Then the Kirchhoff equation (5) takes the form

$$
\frac{d h}{d t}+2 \gamma_{3} h+\omega^{2} \int_{0}^{t} h(\tau) d \tau=-2 \alpha_{0}\left(u^{*}+u\right)+\gamma_{3} f
$$

To derive the evolution equation for the variables (8), we proceed as follows. Write the Heisenberg equations for the corresponding spin components with the standard notation $\omega_{0} \equiv \mu H_{0} / \hbar$ for the Zeeman frequency. Decouple the double spin correlators in the manner described by ter Haar [15], in order to preserve the terms containing the homogeneous spin-spin relaxation $\gamma_{2} \equiv T_{2}^{-1}$, which can be done by using second-order perturbation theory. For generality, we may also include the term describing the spin-lattice relaxation $\gamma_{1} \equiv T_{1}^{-1}$. These steps are known and clear. The most difficult problem is how to treat the local spin fields

$$
\begin{gathered}
\delta_{i}=\frac{1}{\hbar} \sum_{j(\neq i)}^{N}\left\langle\frac{3}{2} a_{i j} S_{j}^{z}+c_{i j} S_{j}^{+}+c_{i j}^{*} S_{j}^{-}\right\rangle, \\
\varphi_{i}=-\frac{2}{\hbar} \sum_{j(\neq i)}^{N}\left\langle b_{i j} S_{j}^{+}+c_{i j} S_{j}^{z}\right\rangle,
\end{gathered}
$$


caused by the dipole interactions

$$
\begin{gathered}
a_{i j}=\frac{\mu^{2}}{r_{i j}^{3}}\left(1-3 \cos ^{2} \vartheta_{i j}\right), \\
b_{i j}=-\frac{3 \mu^{2}}{4 r_{i j}^{3}} \sin ^{2} \vartheta_{i j} \exp \left(-i 2 \varphi_{i j}\right), \\
c_{i j}=-\frac{3 \mu^{2}}{4 r_{i j}^{3}} \sin ^{2}\left(2 \vartheta_{i j}\right) \exp \left(-i \varphi_{i j}\right),
\end{gathered}
$$

where $\vartheta_{i j}$ and $\varphi_{i j}$ are the spherical angles of $\vec{n}_{i j}$. Note that in a uniform approximation the local fluctuating fields (10) are zero because of the properties of the dipole interactions (11). To get a closed set of equations, at the same time retaining the information on the presence of fluctuating fields (10), we may replace the latter by stochastic fields, $\delta_{i} \rightarrow$ $\varphi_{0}, \varphi_{i} \rightarrow \varphi$, the first of which, in compliance with (10), is real and the second is complex. The distribution of these random fields is such that the averaging over it, which we shall denote by $\langle\langle\ldots\rangle\rangle$, gives

$$
\left\langle\left\langle\varphi_{0}\right\rangle\right\rangle=\langle\langle\varphi\rangle\rangle=0, \quad\left\langle\left\langle\varphi_{0}^{2}\right\rangle\right\rangle=\frac{1}{2}\left\langle\left\langle|\varphi|^{2}\right\rangle\right\rangle=\gamma_{*}^{2}
$$

where the dispersion $\gamma_{*}$, in accordance with (10), is of the order of $\gamma_{2}$. In this way, for the spin components (8) we obtain the system of stochastic equations

$$
\begin{gathered}
\frac{d u}{d t}=i\left(\omega_{0}-\varphi_{0}+i \gamma_{2}\right) u-i\left(\gamma_{3} h+\varphi\right) s \\
\frac{d s}{d t}=\frac{i}{2}\left(\gamma_{3} h+\varphi\right) u^{*}-\frac{i}{2}\left(\gamma_{3} h+\varphi^{*}\right) u-\gamma_{1}(s-\zeta), \\
\frac{d|u|^{2}}{d t}=-2 \gamma_{2}|u|^{2}-i\left(\gamma_{3} h+\varphi\right) s u^{*}+i\left(\gamma_{3} h+\varphi^{*}\right) s u
\end{gathered}
$$

The structure of (13) is transparent: $\gamma_{3} h+\varphi$ is the total effective field acting on spins; $h$ is the resonator field defined by $(9) ; \varphi_{0}$ and $\varphi$ model random local fields with a distribution whose particular form is not important since all we need is the property (12). If $\varphi_{0}$ and $\varphi$ were absent, then (13) would be reduced to the Bloch equation.

To consider the case of pure spin superradiance, the initial conditions for the system of equations (9) and (13) are to be taken as 


$$
h(0)=0, \quad u(0)=0, \quad s(0)=z_{0} .
$$

The electromotive force $E_{f}=E_{0} \cos \omega t$ in (5) corresponds to the resonance mode of the thermal Nyquist noise. The driving force in (6) is

$$
f=f_{0} \cos \omega t ; \quad f_{0} \equiv \frac{c \mu E_{0}}{n A_{c} \hbar \gamma_{3}^{2}} .
$$

The system of equations (9) and (13) can be solved by a method [16] combining the guiding-center approach [17] and the method of averaging [18]. The idea is straightforward: First, we classify the variables onto fast and slow. To this end, we take into account the usual inequalities $\gamma_{1} \ll \omega_{0}, \gamma_{2} \ll \omega_{0}, \gamma_{3} \ll \omega$, and consider the quasiresonance case, when $|\Delta| \ll \omega_{0}$, where $\Delta \equiv \omega-\omega_{0}$ is detuning. Thence we notice right away that the variables $h$ and $u$ can be treated as fast, while $s$ and $|u|^{2}$ as slow. Keeping the latter as fixed parameters

$$
s=z, \quad|u|=v,
$$

we get for the fast variables a system of linear equations, which, therefore, is not too difficult to solve. The found solutions for fast variables are to be substituted into the equations for slow variables, and the right-hand sides of these equations are to be averaged over the period $2 \pi / \omega_{0}$ of fast oscillations and also over the distribution of stochastic fields. This procedure results in the equations

$$
\begin{array}{r}
\frac{d z}{d t}=g \gamma_{2} w-\gamma_{1}(z-\zeta)-\gamma_{f} z, \\
\frac{d w}{d t}=-2 \gamma_{2} w-2 g \gamma_{2} w z+2 \gamma_{f} z^{2}
\end{array}
$$

for the slow variables, where

$$
w \equiv v^{2}-\frac{2 \gamma_{*}^{2}}{\omega_{0}^{2}} z, \quad g \equiv \alpha_{0}\left(\frac{\gamma_{3}}{\gamma_{2}}\right) \frac{\pi\left(\gamma_{2}-\gamma_{3}\right)^{2}}{\left(\gamma_{2}-\gamma_{3}\right)^{2}+\Delta^{2}}
$$

and the attenuation

$$
\gamma_{f} \equiv \frac{f_{0}^{2} \gamma_{3}^{4}}{32 \omega_{0}^{2}\left(\Delta^{2}+\gamma_{2}^{2}\right)}\left\{\left(1+\frac{8 \pi^{2}}{3}\right) \gamma_{2}-2 \pi \Delta+\right.
$$




$$
\left.+\frac{\omega_{0} z}{\Delta^{2}+\gamma_{2}^{2}}\left[(\alpha-2 \pi \beta)\left(\Delta^{2}-\gamma_{2}^{2}\right)+2 \gamma_{2} \Delta(\beta+2 \pi \alpha)\right]\right\}
$$

in which

$$
\alpha \equiv \alpha_{0}\left(\frac{\gamma_{3}}{\omega_{0}}\right) \frac{\pi\left(\gamma_{2}-\gamma_{3}\right)^{2}}{\left(\gamma_{2}-\gamma_{3}\right)^{2}+\Delta^{2}}, \quad \beta \equiv \alpha_{0}\left(\frac{\gamma_{3}}{\omega_{0}}\right) \frac{\pi\left(\gamma_{2}-\gamma_{3}\right) \Delta}{\left(\gamma_{2}-\gamma_{3}\right)^{2}+\Delta^{2}}
$$

is due to the action of the driving field (15).

The amplitude of the electromotive force related to the thermal Nyquist noise [19], at temperature $T$ satisfying the inequality $\hbar \omega \ll k_{B} T$, is given by $E_{0}^{2}=\gamma_{3} R k_{B} T / \pi$. Whence for the amplitude of the driving field (15) we have

$$
f_{0}^{2}=\frac{8 \alpha_{0} k_{B} T}{\pi \hbar \gamma_{3} N}
$$

Let us accept the values of parameters characteristic of experiments [7-11] with proton spins: $\quad \omega_{0} \sim \omega \sim 10^{8} \sec ^{-1}, \gamma_{1} \sim 10^{-5} \sec ^{-1}, \gamma_{2} \sim 10^{5} \sec ^{-1}, \gamma_{3} \sim 10^{6} \sec ^{-1}, T \sim 0.1 K$, and $N \sim 10^{23}$. Then $f_{0} \sim 10^{-10}$ and the thermal attenuation (18) is $\gamma_{f} \sim 10^{-16} \sec ^{-1}$. Such an insignificant quantity, of course, plays no role, as compared to all other damping parameters, and has to be neglected in (17).

This result shows, in agreement with Bloembergen and Pound [1], that the Nyquist noise of resonator can never produce the initial thermal relaxation, thus, cannot originate the pure spin superradiance.

Ommitting in (17) the negligibly small $\gamma_{f}$ and taking into account that $\gamma_{1} \ll \gamma_{2}$, we come to

$$
\frac{d z}{d t}=g \gamma_{2} w, \quad \frac{d w}{d t}=-2 \gamma_{2} w(1+g z)
$$

According to (14), the initial conditions are $z(0)=z_{0}$ and $v(0)=0$. Equations in (20) are exactly integrable yielding

$$
\begin{gathered}
z=\frac{\gamma_{0}}{g \gamma_{2}} \tanh \left(\frac{t-t_{0}}{\tau_{0}}\right)-\frac{1}{g}, \\
v^{2}=\left(\frac{\gamma_{0}}{g \gamma_{2}}\right)^{2} \operatorname{sech}^{2}\left(\frac{t-t_{0}}{\tau_{0}}\right)+\frac{2 \gamma_{*}^{2}}{\omega_{0}^{2}} z ;
\end{gathered}
$$


here $\gamma_{0}$ is the radiation width given by

$$
\gamma_{0}^{2}=\Gamma_{0}^{2}-2\left(g \gamma_{2}\right)^{2} \varepsilon_{*} z_{0},
$$

where

$$
\Gamma_{0} \equiv \gamma_{2}\left(1+g z_{0}\right), \quad \varepsilon_{*} \equiv\left(\frac{\gamma_{*}}{\omega_{0}}\right)^{2}
$$

the radiation time $\tau_{0}=\gamma_{0}^{-1}$; and the delay time is

$$
t_{0}=\frac{\tau_{0}}{2} \ln \left|\frac{\gamma_{0}-\Gamma_{0}}{\gamma_{0}+\Gamma_{0}}\right| .
$$

The criterion for the occurrence of spin superradiance is the validity of the inequalities

$$
0<t_{0}<\infty, \quad \tau_{0}<T_{2}
$$

Invoking (22) and (23) and bearing in mind that $\varepsilon_{*} \ll 1$, we find that (24) is equivalent to

$$
z_{0}<z_{p} \equiv-\frac{2}{g}, \quad \varepsilon_{*}>0
$$

As far as $\left|z_{0}\right|<1 / 2$, the first of the inequalities (25) requires that $g \geq 4$. In this way, the pure spin superradiance occurs when the initial spin polarization $z_{0}$ is negative, with an absolute value surpassing the threshold $\left|z_{p}\right|=2 / g$, when the coupling of the spin system with a resonator is sufficiently strong, $g \geq 4$, and if there exist local random fields with a nonzero dispersion $\gamma_{*}>0$.

To emphasize the crucial importance of the local fields, let us notice that if one puts $\varepsilon_{*} \rightarrow 0$, then $\gamma_{0} \rightarrow\left|\Gamma_{0}\right|$ and $\left|t_{0}\right| \rightarrow \infty$. That is, without these fields the pure spin superradiance is impossible. To make the essential dependence of the delay time on $\varepsilon_{*}$ apparent, we may write (23) for the case of strong coupling, when $g\left|z_{0}\right| \gg 1$, then

$$
t_{0} \simeq \frac{T_{2}}{2 g\left|z_{0}\right|} \ln \left|\frac{2 z_{0}}{\varepsilon_{*}}\right|
$$

From here it is evident that $t_{0} \rightarrow \infty$ as $\varepsilon_{*} \rightarrow 0$. For the parameters typical of experiments [7-11] we have $t_{0} \sim 10^{-6}-10^{-5} \mathrm{sec}$. So, this is the local random fields that are responsible for starting the process of self-organization leading to the pure spin superradiance. 
One more question is worth answering: Which part of the local fields is more important for initiating the pure spin superradiance? Recall that the stochastic fields entering into the evolution equations (13) are related to two types of local fields defined in (10). As follows from (13), the term $\delta_{i}$ in (10), corresponding to $\varphi_{0}$, only shifts the rotation frequency, while the term $\varphi_{i}$, corresponding to $\varphi$, starts moving the spin $z$-component even when the resonator feed back field $h$ is yet absent. The term $\varphi_{i}$ in (10) is due to the dipole interactions $b_{i j}$ and $c_{i j}$ defined in (11). These interactions, in the theory of magnetic resonance [14], are called nonsecular interactions, as compared to the secular interaction $a_{i j}$. The initial motion of spins, when $u(0)=0$ and $h(0)=0$, is due solely to the action of nonsecular interactions. This conclusion is in agreement with computer simulations $[4,20]$ for small and mesoscopic spin systems with $N \sim 10-10^{3}$. Thus, we are in a position to give the final answer to the question posed in this paper:

The pure spin superradiance in a nonequilibrium system of polarized nuclear spins can be originated only by local fields due to nonsecular dipole interactions. The thermal Nyquist noise of resonator plays no role in this process.

I would like to express my sincere gratitude to R.Friedberg, S.R.Hartmann, and J.T.Manassah for useful discussions and helpful advises, as well as for their kind hospitality during my visits to the Columbia University and City University of New York. Financial support from the Natural Sciences and Engineering Research Council of Canada is appreciated. 


\section{REFERENCES}

[1] N.Bloembergen and R.Pound, Phys. Rev. 95, 8 (1954).

[2] R.H.Dicke, Phys. Rev. 93, 99 (1954).

[3] R.Friedberg and S.R.Hartmann, Phys. Rev. A 10, 1728 (1974).

[4] T.S.Belozerova, V.K.Henner, and V.I.Yukalov, Phys. Rev. B 46, 682 (1992).

[5] P.V.Zinoviev, V.V.Samartsev, and N.B.Silaeva, Laser Phys. 1, 1 (1991).

[6] P.Bösiger, E.Brun, and D.Meier, Phys. Rev. A 18, 671 (1978).

[7] J.F.Kiselev, A.F.Prudkoglyad, A.S.Shumovsky, and V.I.Yukalov, Mod. Phys. Lett. B 1, 409 (1988).

[8] Y.F.Kiselev, A.F.Prudkoglyad, A.S.Shumovsky, and V.I.Yukalov, J. Exp. Theor. Phys. 67, 413 (1988).

[9] Y.F.Kiselev, A.S.Shumovsky, and V.I.Yukalov, Mod. Phys. Lett. B 3, 1149 (1989).

[10] N.A.Bazhanov, D.S.Bulyanitsa, A.I.Zaitsev, A.I.Kovalev, V.A.Malyshev, and E.D.Trifonov, J. Exp. Theor. Phys. 70, 1128 (1990).

[11] L.Reichertz, H.Dutz, S.Goertz, D.Krämer, W.Meyer, G.Reicherz, W.Thiel, and A.Thomas, Nucl. Instrum. Methods Phys. Res. A 340, 278 (1994).

[12] V.I.Yukalov, Laser Phys. 2, 559 (1992).

[13] T.S.Belozerova, V.K.Henner, and V.I.Yukalov, Comput. Phys. Commun. 73, 151 (1992).

[14] C.P.Slichter, Principles of Magnetic Resonance (Springer, Berlin, 1980).

[15] D. ter Haar, Lectures on Selected Topics in Statistical Mechanics (Pergamon, Oxford, 1977).

[16] V.I.Yukalov, Laser Phys. 3, 870 (1993). 
[17] N.G. Van Kampen, Phys. Rep. 124, 69 (1985).

[18] N.N.Bogolubov and Y.A.Mitropolsky, Asymptotic Methods in the Theory of Non-Linear Oscillations (Gordon and Breach, New York, 1961).

[19] V.M.Fain and Y.I.Khanin, Quantum Electronics (Pergamon, Oxford, 1969).

[20] T.S.Belozerova, V.K.Henner, and V.I.Yukalov, Laser Phys. 2, 545 (1992). 